NTUA 45/94

May 94

\title{
TACHYON EFFECTS ON THE 2-DIM BLACK HOLE GEOMETRY
}

\author{
G.A. Diamandis,B.C. Georgalas \\ Athens University,Physics Department, \\ Nuclear and Particle Physics Section, \\ Panepistimiopolis, Kouponia, \\ GR-157 71,Athens, Greece. \\ and \\ E. Papantonopoulos \\ National Technical University \\ Physics Department \\ GR-157 80 Zografou, \\ Athens, Greece.
}

\begin{abstract}
We study solutions of the tree level string effective action in the presence of the tachyon mode.We find that the 2-dim. static black hole is stable against tachyonic perturbations.For a particular ansatz for the tachyon field we find an exact solution of the equations of motion which exhibits a naked singularity.In the case of static fields we find numerically that the full system has a black hole solution, with the tachyon regular at the horizon.
\end{abstract}


Black holes in two-dimensional gravity have received recently a lot of attention, following Witten's identification of a black hole as a string theory background [1, 2, 3]. The two-dimensional dilaton gravity model was subsequently analyzed by many authors as a simple laboratory to study black hole physics [4. The tachyon field, is a physical string mode that appears in the effective two-dimensional string theory, and the question of how the black hole geometry is affected by the presence of the tachyon field is of interest by itself. This question was studied by a number of authors [5, 6, 7, 8] using various approximation schemes, but without conclusive results as to whether the tachyon back reaction destabilizes the black hole geometry.

De Alwis and Lykken [5] found that a static tachyon field does not alter the black hole character of the dilaton gravity solution. They have included in their investigation tachyon back reaction on the metric, but their results are valid in the asymptotic regime where they can ignore $\mathrm{O}\left(T^{2}\right)$ terms. Using a similar approximation, Rama [6] found tachyon solutions but with a singular scalar curvature at the horizon. Non-singular static solutions were found by the authors of ref. [7], by solving the tachyon equation in the dilaton gravity background.

In this paper we undertake a more carefull and detailed study of the tachyon effects to the dilaton gravity black hole solution. We shall first see that the black hole solution is stable against small perturbations of the tachyonic field. We will show that, if one considers the tachyon equation in a static black hole background, then a small variation of the tachyon field by $\delta T$ does not develop any growing mode, and therefore the black hole is stable.

Of course a more interesting situation arises when tachyon back reaction is considered. In this case the main difficulty is the non linear nature of the coupled differential equations of motion. Working in light cone coordinates and assuming that the tachyon field is a function of only $x^{+}$or $x^{-}$then an exact solution can be found. This is possible because the dilaton field decouples from the tachyon equation which in return can be solved. We attribute this decoupling to a preservation of an $\rho-\phi$ symmetry [9]. The characteristic of this solution is that the tachyon field is singular at some finite $x^{+}$and since the scalar curvature is proportional to the tachyon field, it is also a physical singularity. This singularity is a naked singularity, because as one can easily see, there is no horizon to cover it. It is interesting to note, that this naked singularity remains stable against small perturbations of the 
tachyonic field.

It is difficult to get any other exact solution for different ansätze of the tachyonic field. A considerable simplification arises if all the fields are static. In this case we end up with a simplest system of coupled differential equations. This system can be studied numerically. As we shall discuss in the following, the numerical solution exhibits a clear black hole behaviour with a tachyon field regular at the horizon. The main characteristic of this static solution is that the physical singularity occurs at the strong coupling regime, where both the tachyon and dilaton fields are singular. Such a picture is supported by an analytic expansion procedure in which the expansion parameter plays the role of the mass in Callan et.al. [10]. In the case of $T=0$ in the first order in $\mu$ we get similar results as in [5], while from the second order in $\mu$ we start recognizing the features of the numerical solution.

The action of the dilaton tachyon system coupled to gravity in two dimensions is

$$
S=\frac{1}{2 \pi} \int d^{2} x \sqrt{-g}\left\{e^{-2 \phi}\left[R+4(\nabla \phi)^{2}-(\nabla T)^{2}-V(T)+4 \lambda^{2}\right]\right\}
$$

where $\mathrm{V}(\mathrm{T})$ is the tachyon potential. There are ambiguities of the tachyon potential in string theory [11], but in our discussion quadratic configurations for the tachyon fields will be considered. The equations of motion resulting from the action (11) are

$$
\begin{gathered}
\square \phi-(\nabla \phi)^{2}+\frac{1}{4} R-\frac{1}{4}(\nabla T)^{2}+\frac{1}{2} T^{2}+\lambda^{2}=0 \\
\square T-2(\nabla \phi)(\nabla T)+2 T=0 \\
\square \phi-2(\nabla \phi)^{2}+T^{2}+2 \lambda^{2}=0
\end{gathered}
$$

Using the dilaton eq.(2) the metric equation (四) becomes

$$
\square \phi+\frac{1}{2} R-\frac{1}{2}(\partial T)^{2}=0
$$

The above equations can be most easily analyzed if we work in the conformal gauge

$$
g_{\mu \nu}=e^{2 \rho} n_{\mu \nu} \quad, \quad n_{\mu \nu}=\operatorname{diag}(-1,1)
$$


Using also light-cone coordinates $x^{ \pm}=x^{0} \pm x^{1}$, equations (2),(3) and (5) become

$$
\begin{gathered}
\partial_{+} \partial_{-} \phi-\left(\partial_{+} \phi\right)\left(\partial_{-} \phi\right)-\frac{1}{2} \partial_{+} \partial_{-} \rho-\frac{1}{4}\left(\partial_{+} T\right)\left(\partial_{-} T\right)-\frac{1}{8} e^{2 \rho}\left(T^{2}+2 \lambda^{2}\right)=0 \\
\partial_{+} \partial_{-} T-\left(\partial_{+} \phi\right)\left(\partial_{-} T\right)-\left(\partial_{-} \phi\right)\left(\partial_{+} T\right)-\frac{1}{2} e^{2 \rho} T=0 \\
\partial_{+} \partial_{-} \phi-\partial_{+} \partial_{-} \rho-\frac{1}{2}\left(\partial_{+} T\right)\left(\partial_{-} T\right)=0
\end{gathered}
$$

and in addition we have two constraints

$$
\partial_{ \pm} \partial_{ \pm} \phi-2\left(\partial_{ \pm} \phi\right)\left(\partial_{ \pm} \rho\right)-\frac{1}{2}\left(\partial_{ \pm} T\right)\left(\partial_{ \pm} T\right)=0
$$

In the case of $T=0$ we get the familiar Witten's solution which in the conformal gauge is 10

$$
e^{-2 \phi}=e^{-2 \rho}=\frac{M}{\lambda}-\lambda^{2} x^{+} x^{-}
$$

and for the metric

$$
d s^{2}=-\frac{1}{2}\left(\frac{d x^{+} d x^{-}}{\frac{M}{\lambda}-\lambda^{2} x^{+} x^{-}}\right)
$$

where $\mathrm{M}$ is the black hole mass. The singularity is at $x^{+} x^{-}=\frac{M}{\lambda^{3}}$ and the horizon at $x^{+} x^{-}=0$.

In order to study the black hole stability under small tachyon perturbations we define $\tilde{T}=e^{-\phi} T$ to make the tachyon kinetic terms canonical, and assume

$$
\tilde{T}=\tilde{T}_{0}+\delta \tilde{T}
$$

Then the tachyon equation (7) becomes

$$
\partial_{+} \partial_{-}(\delta \tilde{T})-\left[\left(\partial_{+} \phi\right)\left(\partial_{-} \phi\right)-\partial_{+} \partial_{-} \phi+\frac{1}{2} e^{2^{\rho}}\right](\delta \tilde{T})=0
$$

Using the static solution (10) and defining the space-time coordinates

$$
\begin{aligned}
r & =\ln \left(\frac{M}{\lambda}-\lambda^{2} x^{+} x^{-}\right) \\
t & =-\ln \left(-\frac{x^{-}}{x^{+}}\right)
\end{aligned}
$$


we get

$\partial_{t}^{2}(\delta \tilde{T})-A^{2}(r) \partial_{r}^{2}(\delta \tilde{T})-\frac{M}{\lambda} e^{-r} A(r) \partial_{r}(\delta \tilde{T})+\frac{1}{4 \lambda} A(r)\left(\frac{\lambda^{2}-2}{\lambda}+M e^{-r}\right)(\delta \tilde{T})=0$

with $A(r)=\left(1-\frac{M}{\lambda} e^{-r}\right)$. Separating variables with $\delta \tilde{T}=\tilde{T}(t) \tilde{R}(r)$, then the space dependent equation becomes

$$
\frac{d^{2} \tilde{R}}{d \xi^{2}}+\left(E^{2}-U(\xi)\right) \tilde{R}=0
$$

with $\xi=r+\ln A$, and $U(\xi)$ a complicated function of $\xi$. For $\lambda^{2} \geq 2$ the potential is positive and this implies that the corresponding eigenvalues $\mathrm{E}$ are real and positive, therefore, there are not growing modes for the tachyon disturbances. This means that the static black hole is stable against small tachyon perturbations.In [7] an equation similar to eq.(14) was solved and a nonsingular solution was found. According then to our previous discussion this tachyon hair is stable against small perturbations of the tachyon field. Of course the stability found above is in agreement with the results of [14], where time dependent tachyon perturbations in the black hole solution were discussed. Nevertheless the value of the tachyon field is large near the singularity [2], therefore the back reaction of the tachyon into the gravity dilaton system cannot be ignored.

In an attempt to solve the equations (6) - (9) in order to include the back reaction, we assume that the tachyon is a function of $x^{+}$. Then we get the solution

$$
e^{-2 \phi}=e^{-2 \rho}=u_{+}+u_{-}-\frac{x^{-}}{2} \int^{x^{+}}\left(T^{2}+2 \lambda^{2}\right)
$$

where $u_{-}$is linear in $x^{-}$

$$
u_{-}=b_{-}+c_{-} x^{-}
$$

while the $u_{+}$and the tachyon field obey the equations

$$
\begin{gathered}
\partial_{+}^{2} u_{+}+\left(u_{+}+b_{-}\right)\left(\partial_{+} T\right)^{2}=0 \\
T+\frac{1}{2}\left(\partial_{+} T\right) \int^{x^{+}}\left(T^{2}+2 \lambda^{2}\right)-c_{-}\left(\partial_{+} T\right)=0
\end{gathered}
$$

It is worthing to observe, that our choise of $T=T\left(x^{+}\right)$(or similarly $T=$ $T\left(x^{-}\right)$) respects the $\rho-\phi$ symmetry [9] as we can immediatly see from eq.(8). 
A consequence of that,is the "simple" form of the tachyon equation, where there are no metric or dilaton dependent terms. Eq.(19) can be solved and gives

$$
\frac{d T^{2}}{d x^{+}}=-a\left(T^{2}\right)^{\frac{\lambda^{2}}{2}+1} e^{\frac{T^{2}}{4}}
$$

where $a$ is a constant which must be choosen positive for $\mathrm{T}$ to vanish asymptotically.Eq. (18) can be solved for $u_{+}$using the solution for $T^{2}$ from eq.(20). Substituting $u_{+}$and $u_{-}$to eq.(16) we finally get

$$
e^{-2 \phi}=e^{-2 \rho}=C_{1} F\left(1, \frac{\lambda^{2}}{2}+1 ;-\frac{T^{2}}{4}\right)+C_{2}\left(-\frac{T^{2}}{4}\right)^{-\frac{\lambda^{2}}{2}} e^{-\frac{T^{2}}{4}}-\frac{2 e^{-\frac{T^{2}}{4}}}{a\left(T^{2}\right)^{\frac{\lambda^{2}}{2}}} x^{-}
$$

where $C_{1}$ and $C_{2}$ arbitrary constants. To simplify our discussion we choose $\lambda^{2}=2$, but our results are valid for every $\lambda^{2} \geq 2$. The tachyon eq.(20) can be solved for $x^{+}$giving

$$
x^{+}=\frac{1}{4 a} E_{i}\left(-\frac{T^{2}}{4}\right)+\frac{e^{-\frac{T^{2}}{4}}}{a T^{2}}+d
$$

where $\mathrm{d}$ is a constant. Using eq.(22) we get for $e^{-2 \phi}$

$$
e^{-2 \phi}=e^{-2 \rho}=\frac{4 C_{1}}{T^{2}}\left(1-e^{-\frac{T^{2}}{4}}\right)-2 x^{-} \frac{1}{a T^{2}} e^{-\frac{T^{2}}{4}}
$$

where $x^{-}$was shifted by a constant. We can analyze now our results. First of all from eq.(22) we can see that the tachyon becomes infinite at some finite $x_{0}^{+}$. This infinity is a physical singularity as we can see calculating the curvature

$$
R \propto \frac{C_{1} T^{2}}{4 C_{1}\left(1-e^{-\frac{T^{2}}{4}}\right)-\frac{2}{a} x^{-} e^{-\frac{T^{2}}{4}}}
$$

Of course there is also the usual physical singularity of $x^{-}=2 a C_{1}\left(e^{\frac{T^{2}}{2}}-1\right)$ where the denominator of (24) vanishes. The possible event horizon is given by the equations $\partial_{-} \rho=\partial_{+} \rho=0$. From $\partial_{+} \rho=0$ we get $x^{-}=2 a C_{1}\left(\frac{e^{\frac{T^{2}}{4}}}{1+\frac{T^{2}}{4}}-1\right)$ which covers the singularity at $x^{-}$, while $\partial_{-} \rho=0$ is satisfied only when $T^{2} \rightarrow$ $\infty$. Therefore, the strong coupling regime created by the tachyon infinity is a naked singularity (see Fig.1). 
The question now is, if this new solution we found is stable under small perturbations of the tachyon field. We follow a similar procedure as in the static case. In the tachyon eq.(12) we substitute the solution for $\phi$ from eq. (23).In some appropriate variables $\mathrm{x}$ and $\mathrm{t}$ we find the wave equation

$$
\left(\partial_{t}^{2}-\partial_{x}^{2}\right)(\delta \tilde{T})+V(x, t)(\delta \tilde{T})=0
$$

where $\mathrm{V}(\mathrm{x}, \mathrm{t})$ is a complicated potential, a function of both $\mathrm{x}$ and $\mathrm{t}$. One can show that the potential remains positive and bounded for every $t$, and therefore for $\delta \tilde{T}$ disturbance only oscillatory modes are expected.

What we found is an exact solution which represents a naked singularity. Stability arguments tell us that this solution is stable against small tachyon perturbations. The characteristic of this solution is that it preserves an $\rho-\phi$ symmetry. This symmetry is usefull at the semi classical level because it helps in the solution of the semi-classical dilaton equation of motion [12, 9]. It would be interesting to see of what happens to the geometry if we include to our exact solution the one loop correction of the tachyon field [13]. Being unable to get any other exact solution,we restrict the dilaton field to be static,that is a function of $x^{+} x^{-}$.If we make the following change of variables in the equations of motion

$$
\begin{gathered}
x=\ln \left(-x^{+} x^{-}\right) \\
t=\ln \left(-\frac{x^{+}}{x^{-}}\right)
\end{gathered}
$$

we find that the consistency of the system of eqs (6) - (9) inforces all the other fields to be static and the equations of motion become

$$
\begin{gathered}
T^{\prime \prime}-2 \phi^{\prime} T^{\prime}+\frac{1}{2} e^{2 \rho+x} T=0 \\
\phi^{\prime \prime}-2\left(\phi^{\prime}\right)^{2}+\frac{1}{4} e^{2 \rho+x}\left(T^{2}+2 \lambda^{2}\right)=0 \\
\phi^{\prime \prime}-\rho^{\prime \prime}-\frac{1}{2}\left(T^{\prime}\right)^{2}=0 \\
\phi^{\prime \prime}-\phi^{\prime}-2 \phi^{\prime} \rho^{\prime}-\frac{1}{2}\left(T^{\prime}\right)^{2}=0
\end{gathered}
$$

Thus, in the tachyon equation there are explicit dilaton dependent terms which make the solution very difficult. On the other hand equation (29) tell 
us that the $\rho-\phi$ symmetry is not maintained any more.Nevertheless we can solve the system of eqs.(27) -(30) numerically,specifying the initial conditions of the fields and their derivatives. To quarantee the asymptotic flatness of the fields it is sufficient to start with a negative value of the dilaton derivative.For a specific choise of the initial conditions the results are given in Figs (2) and (3).

In Fig.(2) the dilaton field is plotted as a function of the variable $\mathrm{x}$. We see that from the value $\phi=-\infty$, in which it tends linearly in the asymptotic region, is monotonically increasing becoming singular at finite $\mathrm{x}$. The behaviour of the tachyon field is shown in Fig.(3).Asymptotically it tends to zero and it becomes singular at the same $\mathrm{x}$, where the dilaton is infinite. This point is the position of the physical singularity.Comparing eqs (29) end (30) we get

$$
\rho^{\prime}+\frac{1}{2}=\mu e^{2 \phi}
$$

where $\mu$ is a positive intergration constant. Thus $\rho^{\prime}$ varies monotonically from $+\infty$ to $-\frac{1}{2}$. This means that it vanishes only once at some finite $\mathrm{x}$ where both the dilaton and the tachyon fields are regular. Since for the static case $\rho^{\prime}=0$ solves the equations $\partial_{+} \rho=\partial_{-} \rho=0$, this point gives the position of the horizon.

Such a picture is supported by a more formal analysis of the eqs (27) (30). Assume that the dilaton field is a monotonic function of $\mathrm{x}$. Then we can define a function $p(\phi)$ as the first derivative of $\phi, \frac{d \phi}{d x}=p(\phi)$.Defining $\xi=\rho^{\prime}+\frac{1}{2}$ and using eq.(31) the system of equations (27)-(30) become

$$
\begin{gathered}
p^{2} \frac{d^{2} T}{d \phi^{2}}+p \frac{d p}{d \phi} \frac{d T}{d \phi}-2 p^{2} \frac{d T}{d \phi}+\frac{1}{2} e^{2 \xi} T=0 \\
4\left(2 p^{2}-p \frac{d p}{d \phi}\right)-\left(T^{2}+2 \lambda^{2}\right) e^{2 \xi}=0 \\
p \frac{d \xi}{d \phi}-\mu e^{2 \phi}=0 \\
\frac{d p}{d \phi}-2 \mu e^{2 \phi}-\frac{1}{2} p\left(\frac{d T}{d \phi}\right)^{2}=0
\end{gathered}
$$

We are looking for solution analytic in $\mu$

$$
p=p_{0}+\mu p_{1}+\mu^{2} p_{2}+\ldots
$$




$$
\begin{gathered}
\xi=\xi_{0}+\mu \xi_{1}+\mu^{2} \xi_{2}+\ldots \\
T=T_{0}+\mu T_{1}+\mu^{2} T_{2}+\ldots
\end{gathered}
$$

This can be justified by the fact that the Witten's solution is analytic in the black hole mass which is related to the parameter $\mu$ in eq.(31).It is easy to see that the zero order solution is the flat space solution with $T_{0}=0$. The first order system is

$$
\begin{gathered}
\xi_{1}^{\prime}=-2 e^{2 \phi} \quad, \quad p_{1}^{\prime}=2 e^{2 \phi} \\
T_{1}^{\prime \prime}-2 T_{1}^{\prime}+\frac{2}{\lambda^{2}} T_{1}=0
\end{gathered}
$$

Eq.(37) is the linearized tachyon equation which was considered in [5]. Its solution for $\lambda^{2}=2$ is

$$
T_{1}=\left(A_{1}+B_{1} \phi\right) e^{\phi}
$$

where $A_{1}$ and $B_{1}$ are arbitraty constants. The second order solution gives a tachyon of the form

$$
T_{2}=\left(A_{2}+B_{2} \phi\right) e^{\phi}+\left(\Gamma_{2}+\Delta_{2} \phi\right) e^{3 \phi}
$$

with $A_{2}, B_{2}, \Gamma_{2}, \Delta_{2}$ constants. Therefore the tachyon goes to infinity when $\phi$ gets infinite. In can be shown that at any order the tachyon is always a combination of exponentials $e^{\alpha_{i} \phi}$ with $\alpha_{i}>0$. Furthermore the second order solution for the dilaton gives a physical singularity at a finite point,yielding the basic characterists of the numerical solution we have found.

In conclusion, our main result is that in the case of static fields, the tachyon back reaction maintains the black hole structure of the gravity dilaton system. We have seen that by a numerical analysis of the full system without any approximation, while this is also supported by an expansion procedure where we have assume that the the dilaton field is a monotonic function. In our solution, the tachyon is regular at the horizon, while the physical singularity occurs where both the dilaton and tachyon fields become infinite.Also for a non-static tachyon, in a limited case of a particular anzatz, 
we found an exact solution which represents a naked singularity.Finally, when the tachyon field is not back reacted we found that the black hole geometry is stable against tachyon perturbations.

We had benefited by the discussions we had with A.Lahanas in the early stages of this work. We also want to thank C.Bachas,E. Kiritzis,N.Mavromatos

and L. Thorlacius for valuable discussions. Work partially supported dy C.E.E. Science Program SCI-CT92-0792

\section{References}

[1] E. Witten, Phys.Rev. D44 (1991) 314.

[2] E. Mandal, H. Sengupta and S.R. Wadia, Mod.Phys.Lett. A6 (1991) 1685 .

[3] R. Dijkraaf, H. Verlinde and E. Verlinde, Nucl.Phys. B371 (1992) 269.

[4] For a review and references therein see for example S. B. Giddings in the proceedings of the International Workshop of Theoretical Physics, Erice June 1992, and preprint UCSBTH-92-36.

[5] S.P. De Alwis and J. Lykken, Phys.Lett. B269 (1991) 264.

[6] S.K. Rama, Nucl.Phys. B407 (1993) 191.

[7] A. Peet, L. Susskind and L. Thorlacius, Phys.Rev. D48 (1993) 2415.

[8] S. Chaudhuri and D. Minic, Phys.Lett. B312 (1993) 79; N. Marcus and Y. oz, Nucl. Phys. B407 (1993) 429; J. Russo, Phys.Rev. D47 (1993) R4188.

[9] J.G.Russo, L. Susskind and L. Thorlacius, Phys. Rev. D46 (1992) 3444.

[10] C.G. Callan, S.B. Giddings, J.H. Harvey and A. Strominger, Phys.Rev. D45 R1005 (1992).

[11] T. Banks, Nucl.Phys. B361 (1991) 166. 
[12] A. Bilal and C. Callan,Nucl.Phys. B394 (1993) 73; S.P. de Alwis, Phys.Lett. B300 (1993) 330.

[13] Paper in preparation

[14] G. Diamandis, B.C. Georgalas, X. Maintas and N.E. Mavromatos, Phys.Lett. B297 (1992) 67. 